\title{
An Efficient Micro-Mobility Solution for SIP Networks
}

\author{
Dimitra Vali \\ OTE Research \\ Hellenic Telecommunications Organization - OTE S.A. \\ Athens, Greece \\ dvali@oteresearch.gr
}

\author{
Sarantis Paskalis, Alexandros Kaloxylos, Lazaros Merakos \\ Department of Informatics and Telecommunications \\ University of Athens \\ Athens, Greece \\ \{paskalis, agk, merakos\}@di.uoa.gr
}

\begin{abstract}
SIP is evolving as the dominant protocol for multimedia call control in IP networks and is expected to be widely deployed in the near future. Using SIP for supporting mobility in SIPbased networks appears as a very attractive alternative to Mobile IP, taking advantage of existing SIP infrastructure and signaling, while avoiding duplication of functionality. However, existing proposals for supporting mobility with SIP are not efficient in handling micro-mobility and do not cater for all types of traffic.
\end{abstract}

In this paper, we introduce Hierarchical Mobile SIP (HMSIP), for efficient micro-mobility management in SIP environments. HMSIP is a SIP-based scheme that builds on existing IP mobility protocols, aiming at integrating their key concepts in the most beneficial way. Our proposed scheme minimizes the handoff latency and the backbone signaling overhead, while catering for all types of traffic. Moreover, HMSIP can be effectively combined with micro-mobility schemes for QoS flows, producing, thus, a complete micro-mobility solution for SIP flows with QoS guarantees.

\section{Keywords; SIP, micro-mobility, QoS.}

\section{INTRODUCTION}

The explosive growth of mobile phone users worldwide, along with the increasing Internet penetration in human populations, has led to the users' need for accessing high speed Internet data applications via their mobile terminal, while still being able to make high quality voice calls. The widely accepted prediction that data traffic will comprise the vast majority of served traffic in future fixed and mobile networks, is the major drive behind the design of next generation networks based on an all-IP model similar to the Internet.

All-IP networks face among others, the challenges of Quality of Service (QoS) provision, mobility support and their combination. More specifically, users are not willing to compromise the accustomed quality of their voice calls for the sake of data services. IP networks, however, were designed as best-effort networks. Intensive efforts have been made for enhancing Internet with QoS supporting schemes (such as MPLS [1], DiffServ [2], IntServ [3], RSVP [14]) and a major part of the research community is still working on this area.

Moreover, the Internet was initially conceived as a network for serving fixed hosts and no provision was made for supporting mobile users. Mobile IP (MIP) [5] and Mobile IPv6 (MIPv6) [6] were proposed lately for supporting terminal mobility at the network layer. There have also been efforts
[7][8][9] for supporting IP mobility at the application layer using the Session Initiation Protocol (SIP), as an alternative to network-layer mobility. For simplicity reasons, we will refer to these efforts as "Mobile SIP (MSIP)" (in accordance to "Mobile IP (MIP)").

SIP [10] is a simple, text-based, application-layer protocol that is gaining wide acceptance as the call control protocol for multimedia services in IP networks. The Third Generation Partnership Project (3GPP) has already adopted SIP for multimedia call control in next generation networks. While being a call control protocol, SIP can intrinsically handle user mobility, since it uses logical addresses (SIP Uniform Resource Identifiers - SIP URIs) for identifying SIP users, independently of the device they are currently using.

With some minor extensions, SIP can handle terminal mobility as well, taking advantage of existing SIP infrastructure (i.e. SIP proxies, SIP registrars, etc.). In contrast, deploying Mobile IP in SIP networks leads to some extent in a duplicated network functionality and stored user data (e.g. in MIP Home Agent and in SIP Home Registrar). In addition, the use of Mobile SIP could also compensate for the current lack of wide deployment of Mobile IP.

Mobile SIP and Mobile IP perform, however, poorly in high mobility environments. The communication reestablishment after a handoff requires end-to-end signaling exchange, resulting in long update delays and high signaling overhead in the backbone network. The need, therefore, arises for micro-mobility schemes that efficiently handle intradomain handoffs. Micro-mobility schemes constrain mobility related signaling inside the boundaries of the domain, while catering for fast redirection of the data path to the new location of the user. Throughout this paper, we refer to the intra-domain handoff as the handoff of a mobile to a new subnet inside the same administrative domain, resulting in the allocation of a new IP address to the mobile. Inter-domain handoff is defined as the handoff of the mobile to a subnet belonging to a new domain.

Moreover, Mobile IP and Mobile SIP exhibit major deficiencies when handling mobility of resource reserving QoS flows. The mobility related end-to-end signaling exchange results in end-to-end reservation re-establishment, long handoff delays and bandwidth waste through over-reservation. The micro-mobility schemes can facilitate fast local re- 
establishment of reserved resources while preserving the backbone reservations.

In this paper, we introduce a SIP-based scheme for the efficient mobility support inside a SIP domain, named Hierarchical Mobile SIP (HMSIP). Our approach aims at reducing the handoff latency and minimizing the signaling overhead in the backbone network, by restricting intra-domain handoff related signaling inside the roaming domain. HMSIP can be further combined with other efforts for micro-mobility of QoS flows, resulting in a complete solution for mobility of SIP sessions with QoS guarantees.

The remainder of this paper is organized as follows: section 2 discusses prior related work in more detail; section 3 describes the functionality of the HMSIP; section 4 discusses combining HMSIP with mobility schemes for QoS sessions; section 5 assess the proposed scheme, presents conclusions and future work.

\section{RELATED WORK}

Over the past years various network-layer micro-mobility schemes have been suggested, aiming mainly at restricting mobility related signaling inside the domain after an intradomain handoff. Amongst them, Cellular IP [11] and HAWAII [12] use mobile-specific routing modifications inside the access domain. A specialized path-setup protocol is used inside the domain to install soft-state host specific forwarding entries at the domain routers for each roaming mobile. The domain is connected to the Internet via a special gateway, responsible for terminating domain specific signaling.

Other Mobile IP enhancing schemes, such as Hierarchical Mobile IPv6 (HMIPv6) [13] and Mobile IP Regional Registration (MIP-RR) [14], for MIPv6 and MIP respectively, extend the Mobile IP concept into a hierarchy of Mobility Agents inside the domain. The Mobile Host (MH) registers with the nearest mobility agent at the lowest hierarchy level, which in turn registers with its nearest agent at the next hierarchy-level, and so on, up to the Home Agent (Hierarchical Registration). With this technique, when the $\mathrm{MH}$ performs an intra-domain handoff, the registration signaling propagates only up to the local mobility agent. Packets addressed to the $\mathrm{MH}$ travel down the hierarchy, being tunneled from one level to the next.

The network-layer micro-mobility approaches described above result in duplication of functionality and stored data when applied in SIP networks. This is because both SIP and the various micro-mobility protocols have their own mechanism for registration/location update after handoff and their own database for storing location information for the mobile host. Their combined use results, therefore, in unnecessary protocol and stored data overhead.

Apart from network-layer approaches, application- layer schemes have also been developed for supporting mobility in an IP environment. Motivation and description of Mobile SIP functionality can be found in [7], [8] and [9]. The authors argue that SIP can be used to provide terminal mobility to Internet multimedia applications, proposing appropriate SIP extensions to the base SIP specification [10]. According to this approach,
SIP on-going sessions need to be re-established after a handoff to a new access router. End-to-end SIP session establishment messages are exchanged, containing the new IP address of the $\mathrm{MH}$ and an updated session description [15] for the on-going session, enabling the Correspondent Host $(\mathrm{CH})$ to send all subsequent data to the new location of the $\mathrm{MH}$.

Mobile SIP is not, however, well suited for intra-domain handoffs, since it entails end-to-end signaling exchange for IP address update after handoff. For this reason, enhancements to Mobile SIP for fast intra-domain handoff are further suggested in [8], targeted, however, for handling RTP (Real Time Protocol) [16] flows only. A hierarchical SIP-based registration scheme is proposed that accelerates the registration procedure by reducing backbone SIP signaling. The authors suggest the use of a SIP server inside the domain terminating the handoff related SIP messages. The authors also propose the use of an RTP translator [16], to redirect downlink RTP traffic to the new location of the user, which implies, several modifications to the standard RTP discard behavior of the $\mathrm{CH}^{1}$.

Another effort for handling micro-mobility is presented in [17]. The Intra-Domain Mobility Protocol (IDMP) is introduced that can interwork with either Mobile IP or Mobile SIP inter-domain mobility protocols. IDMP, similar to the concepts of MIP-RR and HMIPv6, allocates a local and a global care-off address (CoA) to each roaming mobile inside the domain, while using a Mobility Agent (MA) for handling intra-domain mobility. IDMP specifies a location update message, used for registering the MH's local CoA with the designated MA, and the respective location reply message that communicates the allocated global CoA to the MH. IDMP is a network-layer micro-mobility protocol, and, as such, shares the same deficiencies with the various network-layer based schemes described previously.

The micro-mobility scheme proposed in this paper is based on SIP and attempts to integrate the benefits of the various aforementioned micro-mobility approaches, resulting in reduced handoff delay and backbone signaling overhead. HMSIP reuses the existing SIP infrastructure (proxies, servers, registrars) avoiding, thus, the duplication of functionality and stored data that appears in other schemes. Moreover, HMSIP caters for all types of traffic and sets no requirements to the $\mathrm{CH}$, apart from supporting standard SIP procedures. Finally, we argue that HMSIP facilitates efficient mobility of QoS flows with reserved resources.

HMSIP and its relation with the various mobility schemes are depicted in Figure 1.

\footnotetext{
${ }^{1}$ When a moving terminal acquires a new IP address, the CH's RTP module considers received RTP packets, carrying the same SSRC identifier (Synchronization Source Identifier) identifying RTP sessions- and different source IP addresses, as looped packets and discards those arriving from the new IP address [16].
} 


\section{HMSIP}

\section{A. Overview}

HMSIP follows the general regional registration approach found in various proxy-agent micro-mobility schemes (e.g. MIP-RR, HMIPv6, IDMP) and builds on the SIP Hierarchical Registration proposed in [8]. Moreover, HMSIP introduces the concept of a SIP Mobility Agent (SIP MA), a domain border entity responsible for locally handling intra-domain mobility, enabling thus fast intra-domain handoffs. The SIP MA terminates the handoff related signaling inside the domain and performs fast data path redirection to the current location of the mobile.

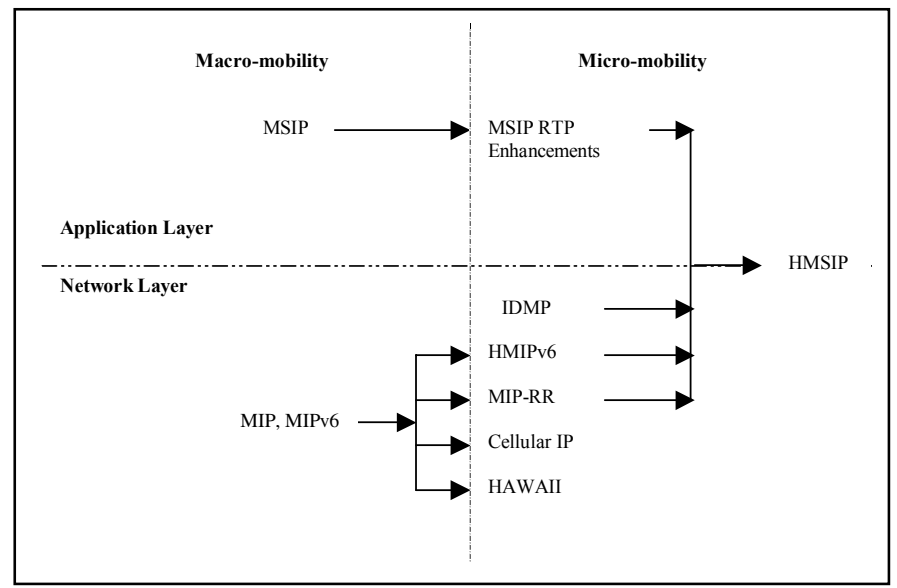

Figure 1 Protocol relationships

Similarly to other micro-mobility approaches, HMSIP allocates two IP addresses to the MH, a Local Address (LA) and a global Domain Address (DA). The LA is an IP address reflecting the current point of attachment of the $\mathrm{MH}$. and is allocated to the MH by the serving access router. A new LA is allocated to the $\mathrm{MH}$ each time it performs an intra-domain handoff. The DA is a globally routable IP address, that uniquely identifies the $\mathrm{MH}$ for the whole duration of its roaming inside the same access domain. Each mobile is allocated a different DA by the SIP MA, which has a pool of globally routable IP addresses associated with it.

The SIP MA (Figure 2) is a domain border router, enhanced with the functionality of a SIP Proxy/B2BUA [10] and a SIP Registrar. The SIP MA is responsible for maintaining and managing a database with soft state mappings between the SIP URI, the DA and the LA for each mobile that roams inside the domain. Soft states allow an allocated DA to be released when the $\mathrm{MH}$ visits another domain or goes down for a long time.

For simplicity reasons, we assume in the following sections that each domain is controlled by a single SIP MA situated at the domain boundary.

\section{B. Mobility Procedures}

HMSIP caters for intra-domain mobility and relies on Mobile SIP for handling inter-domain mobility. In this subsection, we describe in detail the main mobility procedures; namely registration and handover (intra-domain and interdomain).

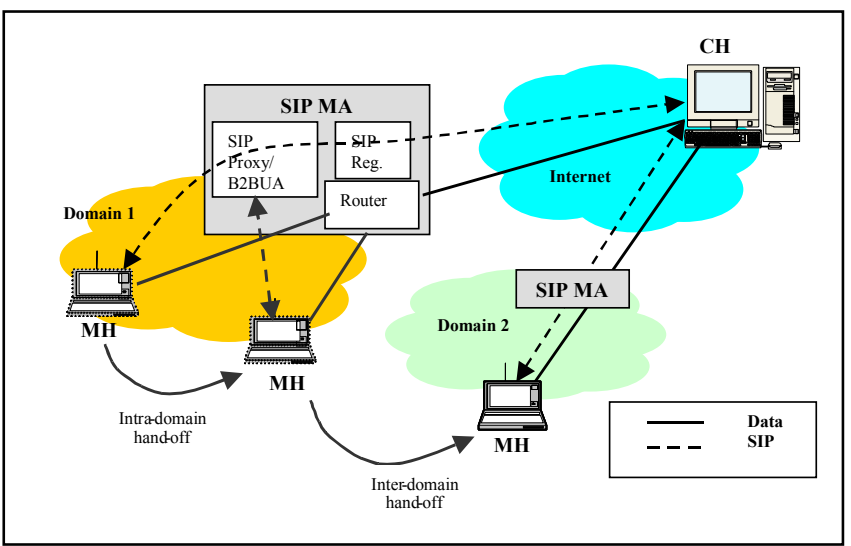

Figure 2 HMSIP architecture

\section{Registration Procedure}

The Registration phase builds upon the SIP Hierarchical Registration proposed in [8], with some modifications and additions.

After powering-on inside a visiting domain, the $\mathrm{MH}$ is allocated a LA from the serving access router, as well as a DA from the serving SIP MA. The new LA and DA should be registered with the MH's visited (Regional Registration) and home (Home Registration) network respectively, as shown in Figure 3.

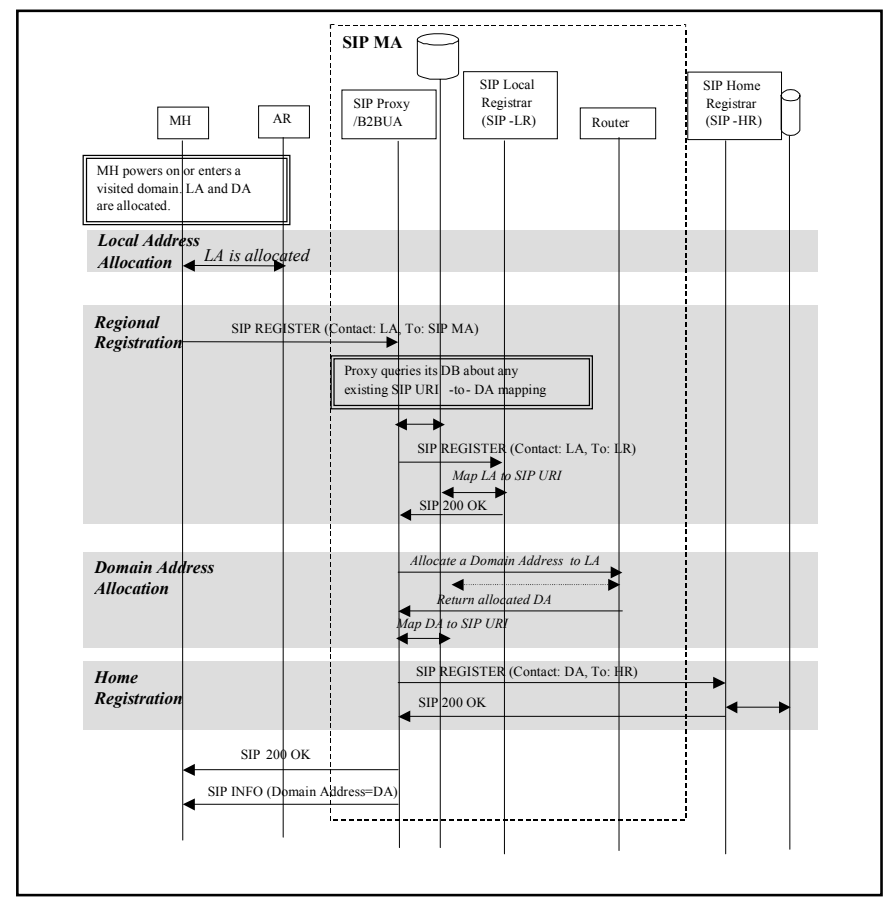

Figure 3 Regional and Home Registration

Specifically, the MH sends a SIP REGISTER message towards its serving SIP MA to register its new location with the visited network. The address of the serving SIP MA is advertised to the $\mathrm{MH}$ by the serving access router and is stored internally in the $\mathrm{MH}$. The Contact field of the REGISTER 
message includes the LA. The SIP Registrar entity of the SIP MA creates a (or updates an existing) record containing the respective LA-to-SIP URI mapping. This completes the Regional Registration procedure.

In case no relative record already exists, regarding the specific SIP URI, the MH is considered as newly arrived to the domain and, as such, is allocated a new DA by the domain SIP MA. The respective mapping between SIP URI, LA and the DA allocated is stored in the Registrar entity of the SIP MA. Periodic Register messages are used for refreshing the stored mappings.

To enable Home Registration, the SIP Local Registrar instructs the SIP proxy/B2BUA entity of the serving SIP MA to initiate a SIP REGISTER message towards the SIP Home Registrar of the MH (Figure 4). The location of the SIP Home Registrar can be retrieved from the SIP URI of the MH. The REGISTER message contains the DA in the Contact field. As a result, the SIP Home Registrar creates a mapping between the SIP URI and the DA of the MH in its database.

Finally, the SIP MA communicates to the MH its allocated DA, including it inside the payload of a SIP INFO message. The DA will be subsequently used as the static address of the $\mathrm{MH}$ inside the roaming domain and will be the source address for all outgoing signaling and data packets.

After a successful Regional and Home Registration, the $\mathrm{MH}$ is able to initiate or accept new SIP calls. Incoming SIP calls towards the $\mathrm{MH}$ are directed to the DA due to the Home Registrar mappings maintenance. Encapsulation of respective SIP signaling for incoming and outgoing SIP calls takes place inside the domain between the SIP MA and the current location (LA) of the MH (Figure 5).

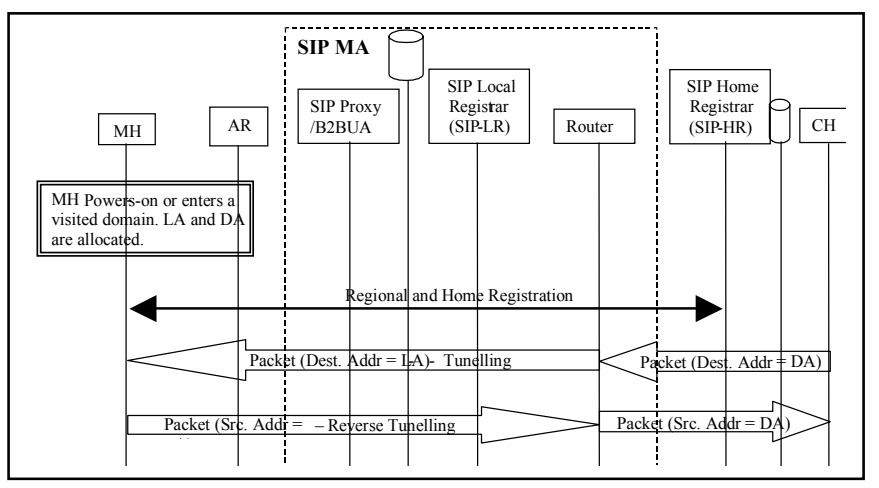

Figure 4 Session establishment and data exchange

Similar to signaling packets, data packets exchanged between the $\mathrm{MH}$ and the $\mathrm{CH}$ use DA as source/destination address outside the domain, while encapsulation to/from LA takes place inside the domain.

\section{Intra-domain handoff procedure}

When the MH hands off to a new access router, it compares the advertised SIP MA address with the internally stored SIP MA address. If the advertised address coincides with the stored one, the $\mathrm{MH}$ is considered to have performed an intra-domain handoff (we have made the simplifying assumption that each domain is controlled by a single SIP MA).
In that case, a new LA is allocated to the MH by the access router, while the DA remains the same. The Regional Registration procedure is triggered (Figure 5), resulting in updating the DA-LA mapping in the HMSIP Agent to reflect the new location of the MH. Home Registration does not take place, since the DA has not changed, allowing for the restriction of the mobility related signaling inside the domain boundaries.

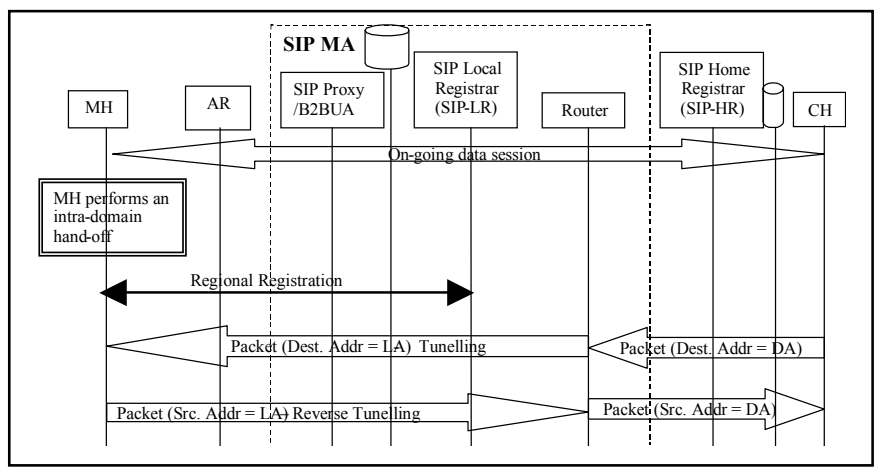

Figure 5 Intra-domain handoff

We note that there is no need for re-establishment of ongoing SIP sessions after an intra-domain handoff. The SIP MA is kept up to date with the new LA of the MH via the Regional Registration procedure and is therefore able to redirect the data path to the new location of the $\mathrm{MH}$.

\section{Inter-domain handoff procedure}

After entering a new domain, the $\mathrm{MH}$ is allocated a LA from the serving Access Router, as well as a DA from the serving SIP MA. Regional Registration and Home Registration procedures take place, as described before.

Following the successful Regional and Home Registration, the session re-establishment (with updated SDP) takes place for on-going SIP sessions, as was initially proposed by the Mobile SIP approach ([7][8][9]). The re-establishment of on-going SIP sessions enables the $\mathrm{CH}$ to be informed about the new location of the $\mathrm{MH}$ and to send subsequent data to the new location. We note that all subsequent SIP signaling should include the ongoing session's ID.

En- and de-capsulation of SIP signaling and data packets takes place at the SIP MA of the roaming domain, as described in detail in the power-on case.

\section{HMSIP AND MOBILITY OF QOS FLOWS}

In QoS-enabled communication, reserved resources are often needed to maintain the QoS guarantees of the flows. In mobility situations, where the IP address of any of the endpoints changes, reservations need to be re-established end-toend [18]. In high mobility environments this results in long handoff delay and significant waste of bandwidth through overreservation.

Several efforts have been made for efficiently handling local mobility of QoS sessions with reserved resources (e.g. [19], [20] and [21], amongst others). The proposals ranged from protocol modification (RSVP modifications to cater for 
mobility) to special mobility aware node introduction to ameliorate limited mobility.

Since HMSIP is similar in concept to other micro-mobility protocols, it retains the benefit of a constant IP address for movement inside the domain. Therefore, no modifications are required for RSVP-enabled routers in the exterior network. The only needed addition would be a mobility enhanced RSVProuter [21] at the border of the HMSIP domain. This router would split the QoS flow into a non-volatile exterior flow (where the end IP addresses do not change) and its complementary interior flow (where the mobile IP address does change, and thus, the reservation needs to be re-established after every handoff).

Collocating the mobility enhanced RSVP router with the SIP MA enhances the micro-mobility of SIP sessions with efficient QoS management.

\section{CONCLUSIONS AND FUTURE WORK}

In this paper, we introduce Hierarchical Mobile SIP (HMSIP) for efficient micro-mobility management inside a SIP domain. HMSIP is based on a hierarchical access domain architecture that minimizes mobility related SIP signaling in the backbone network, by restricting intra-domain handoff related signaling inside the roaming domain. In addition, HMSIP enables fast redirection of the data path to the new user location. It supports intra-domain mobility for all types of traffic and sets no specific requirements to the far end-system, apart from exhibiting standard SIP behavior.

HMSIP is based on SIP, a feature that can be proven to be particularly advantageous in future telecom networks, where SIP deployment is expected to be wide. Mobility management is performed with the reuse of existing SIP infrastructure, enabling the seamless interworking with existing SIP-based mobility proposals. Moreover, HMSIP is also based on the key concepts of Hierarchical Mobile IP schemes and integrates their functionality with the SIP enabled infrastructure.

It is important to note that HMSIP can be effectively combined with hierarchical QoS micro-mobility schemes, enabling efficient micro-mobility support for SIP sessions with reserved resources.

Future work will focus on validating the enhanced performance of HMSIP, comparing it with Mobile SIP, as well as with other micro-mobility approaches.

\section{REFERENCES}

[1] E. Rosen, A. Viswanathan, R. Callon, "Multiprotocol Label Switching Architecture", rfc 3031, January 2001
[2] S. Blake, D. Black, M. Carlson, E. Davies, Z. Wang, W. Weiss, “An Architecture for Differentiated Services”, IETF RFC 2475, December 1998

[3] R. Braden, D. Clark, S. Shenker, "Integrated Services in the Internet Architecture: an Overview", IETF RFC 1633, June 1994

[4] R. Braden, L. Zhang, S. Berson, S. Herzog, S. Jamin, "Resource Reservation Protocol (RSVP)", IETF RFC 2205, September 1997

[5] C. Perkins, "IP Mobility Support", RFC 2002, October 1996.

[6] D. Johnson, C. Perkins, "Mobility Support in Ipv6", Internet Draft (Work in progress), July 2001

[7] E. Wedlund, H. Schulzrinne, "Mobility support using SIP", 2nd ACM/IEEE International Conference on Wireless and Mobile Multimedia, Seattle, Washington, Aug. 1999

[8] H. Schulzrinne, E. Wedlund, "Application layer mobility using SIP", Mobile Computing and Communications Review, Volume 4, Number 3

[9] A. Dutta, F. Vakil, J.-C. Chen, M. Tauil, S. Baba, N. Nakajima, and H. Schulzrinne, "Application layer mobility management scheme for wireless Internet", In Proc. of IEEE International Conference on Third Generation Wireless and Beyond (3Gwireless'01), (San Francisco, CA), pp. 379-385, May 2001.

[10] J. Rosenberg, H. Schulzrinne, G. Camarillo, A. Johnston, J. Peterson, R. Sparks, M. Handley, E. Schooler, "SIP: Session Initiation Protocol", RFC 3261, June 2002

[11] Campbell, J. Gomez, S. Kim, A. Valko, Wan Chien-Yin, Z. Turanyi, "Design, implementation, and evaluation of cellular IP", IEEE Personal Communications, Vol. 7, August 2000, pp. 42-49

[12] R. Ramjee, T. La Porta, S. Thuel, K. Varadhan, S. Y. Wang, "HAWAII: a domain-based approach for supporting mobility in wide-area wireless networks", Proceedings of the Seventh International Conference on Network Protocols (ICNP) 1999, pp. 283-292

[13] C. Castelluccia, "HMIPv6: A Hierarchical Mobile IPv6 Proposal.", ACM Mobile Computing and Communication Review (MC2R) - April 2000

[14] E. Gustafsson, A. Jonsson, C. Perkins, "Mobile IP Regional Registration", Internet Draft (work in progress), March 2001

[15] M. Handley, V. Jacobson, "SDP: Session Description Protocol", IETF RFC 2327, April 1998

[16] H. Schulzrinne, S. Casner, R. Frederic, V. Jacobson, "RTP: A transport protocol for real-time applications", IETF RFC 1889, Jan. 1996

[17] S. Das, A. Dutta, A. McAuley, A. Misra and S. Das, "IDMP: An IntraDomain Mobility Management Protocol using Mobility Agents, Internet Draft, Work in Progress, January 2000

[18] M. Thomas, "Analysis of Mobile IP and RSVP interactions", Internet Draft, Work in Progress, Feb. 2001

[19] A. Talukdar, B. Badrinath, and A. Acrarya, "MRSVP: A resource reservation protocol for an integrated services network with mobile hosts", The Journal of Wireless Neworks, 2001

[20] C.-C. Tseng, G.-C.Lee, and R.-S.Liu, "HMRSVP: A hierarchical mobile RSVP protocol", International Workshop on Wireless Networks and Mobile Computing (WNMC2001), April 2001

[21] S. Paskalis, A. Kaloxylos, E. Zervas, L. Merakos, "An efficient RSVPMobile IP Interworking Scheme", Journal for Special Topics in Mobile Networks and Applications (MONET), vol. 8, no. 3, June 2003. 